\title{
Upcoming Meetings Related to Alzheimer's Disease*
}

JULY 2005

Conference:

Date:

Location:

Contact:

\section{AUGUST 2005}

Conference:

Date:

Location:

Contact: 13th Annual Alzheimer's Association Dementia Care Conference 26-29 July 2005

Hyatt Regency Chicago Hotel, Chicago, IL, USA

Email: careconference@alz.org

http://alz.org/careconference

20th Biennial Meeting of the International Society for Neurochemistry

21-26 August 2005

Innsbruck, Austria

christiane.riedl@uibk.ac.at

Phone: 435125043715

Fax: 435125043716

http://www.isn-esn2005.org/

\section{SEPTEMBER 2005}

Conference:

Date:

Location:

Contact:

Conference:

Date:

Location:

Contact:

Conference: 9th Congress of the European Federation of Neurological Societies

17-20 September 2005

Congress Centre OMMA of the Megaron-Athens Concert Hall, Athens, Greece efns05@kenes.com

Phone: +41229080488

Fax: +4122732 2850

17 Rue du Cendrier, PO Box

1726, Geneva, CH-1211, Switzerland

http://www.kenes.com/efns2005/

130th Annual Meeting of the American Neurological Association

26-29 September 2005

San Diego, CA, USA

lorijanderson@msn.com

5841 Cedar Lake Road, Suite \#204, Minneapolis, Minnesota, 55416, USA

21st International ADI Conference

${ }^{*}$ Derived from listings at Alzheimer Research Forum (http://www.alzforum.org). 
Date:

Location:

Contact:

\section{OCTOBER 2005}

Conference:

Date:

Location:

Contact:

Conference:

Date:

Location:

Contact:

Conference:

Date:

Location:

Contact:

Conference:

Date:

Location:

Contact:

Conference:

Date:

Location:

Contact:

Conference:

Date:

Location:

Contact:
28 September 2005 - 1 October 2005

Istanbul, Turkey

congress@topkon.com

Phone: +90 2164670647

Fax: +9021646706 51

http://www.alz.co.uk

Regional Symposium on Alzheimer's Disease in the Middle East

1-2 October 2005

Istanbul, Turkey

Robert Friedland, MD - robert.friedland@ case.edu

Phone: 12163681912

Fax: 121636819

http://www.worldeventsforum.com/alz.htm

5th Leonard Berg Symposium: Antecedent Biomarkers for Early \& Preclinical Detection of Alzheimer's

7-8 October 2005

Eric P. Newman Education Center, Washington University School of Medicine, St. Louis, MO, USA

Tom Meuser - Email: meusert@abraxas.wustl.edu

Phone: 314-286-2882

http://alzheimer.wustl.edu/adrc2/BergSymp/Berg2005/Sche dule.htm\#Day1

Genetics of Alzheimer's Disease and Related Disorders

20-21 October 2005

Four Seasons Resort and Club, Irving, TX, USA

Email: Alzheimers2005@UTSouthwestern.edu

Fourth International Congress on Vascular Dementia

20-23 October 2005

Porto, Portugal

Mandy Engel - Email: vascular@kenes.com

Phone: +41 229080488

Fax: +41 227322850

http://www.kenes.com/vascular/

18th Congress of the European College of Neuropsychopharmacology

22-26 October 2005

Amsterdam, Netherlands

secretariat@ecnp.nl

http://www.ecnp.nl/

3rd Canadian Colloquium on Dementia

27-29 October 2005

Ottawa Westin, Ottawa, Ontario, Canada

Email: r.keren@utoronto.ca

http://www.ccd2005.ca 


\section{NOVEMBER 2005}

Conference:

Date:

Location:

Contact:

Conference:

Date:

Location:

Contact:

Conference:

Date:

Location:

Contact:

Conference

Date:

Location:

Contact:

\section{FEBRUARY 2006}

Conference:

Date:

Location:

Contact:

\section{APRIL 2006}

Conference:

Date:

Location:

Contact:

Address
World Congress of Neurology 2005

5-13 November 2005

Sydney, Australia

The Neurological Society of Thailand Division of Neurology, Department of Medicine

-info@thaineurology.org

Phone: 66-22-011-386

Ramatibodi Hospital Mahidol University, Phayathai Road, Bangkok, 10400, Thailand

18th World Congress on Neurology

5-13 November 2005

Sydney Convention \& Exhibition Centre, Sydney, Australia

conference@wcn2005.com

Phone: +61292411478

Fax: +61292513552

GPO Box 2609, Sydney, NSW 2001

http://www.wen2005.com

35th Annual Meeting of the Society for Neuroscience

12-16 November 2005

Washington, DC, USA

info@sfn.org

Phone: 1-202-462-6688

11 Dupont Circle, N.W., Suite 500, Washington, DC, 20036, USA

2nd International Congress on Brain and Behavior

17-20 November 2005

Thessaloniki, Greece

Dr. KN Fountoulakis - kfount@med.auth.gr

Phone: 302310994662

Fax: 302310266570

Fourth Kuopio Alzheimer Symposium

3-4 February 2006

Kuopio, Finland

Tuija Parsons - tuija.parsons@uku.fi

http://www.uku.fi/neuro/AD2006

9th International Geneva/Springfield Symposium on Advances in Alzheimer Therapy 19-22 April 2006

CICG, International Conference Centre, Geneva, Switzerland

Ann Hamilton - Email: ahamilton@ siumed.edu

Southern Illinois University School of Medicine, P.O. Box 19602, Springfield, Illinois, 62794-9602, USA

Phone: (217) 545-7711

Fax: (217) 545-4413 